steplike double or single wedge in cross section, the appearance is very striking, each thickness glowing with its particular color.

To produce a continuous wedge effect, the width of the strips should be gradually decreased to the center of the field. Since stress is greatest where the cross-section is least, the higher orders of colors appear nearer the center.

5. Compensation Effects.-If a plate quartz, cut parallel to the axis and showing the warm red between crossed nicols, is placed with its axis parallel to the lines of strain of the celluloid strip, the succession of colors is red, orange, yellow, green, blue or retrograde. The quartz effect is thus gradually more and more neutralized by the celluloid strip as its strain increases. If the axis of the quartz plate is at right angles to the lines of strain, the order is red, purple, blue, green, yellow or direct. The quartz and celluloid effect coincide in sign. Hence the ray vibrating in the direction of the lines of stress corresponds to the ordinary ray in quartz, which from the positive character of the wave surface, is the swifter. It follows that the ray vibrating parallel to the lines of stress of the celluloid strip moves with greater velocity than the ray vibrating normally to this direction. In other words the extraordinary ray is swifter and the wave surface of strained celluloid is negative: for on inclining the celluloid strip around the lines of stress as an axis, the succession of colors (due to increasing thickness) is direct in order; whereas on inclining the strip around an axis normal to the lines of stress the succession is actually retrograde, showing that the optic axis is being rapidly approached, where double refraction ceases in spite of thickness.

Similarly if a quarter wave plate of mica is inserted with the effective axis parallel to the lines of stress, the order of colors for crossed nicols is bluish, dark (neutral, compensation), bluish, yellowish, etc. (excessive celluloid effect), or clearly retrograde. If inserted with the axis normal to the lines of stress the succession of color is bluish, yellowish, red, purple, blue, etc., or direct, all of which admits of the same interpretation as in the case of quartz.

An equally interesting compensation is obtained by crossing two similarly stretched strips of celluloid at right angles. In this case the area where the strips overlap is quite neutral (dark between crossed nicols) while the four non-duplicated areas, extending outward from the square center, are vividly colored. The faster ray in one strip becomes the slower in the other, and vice versa.

My thanks are due to Professor Barus, who suggested these experiments, for his aid throughout the whole course of the work.

\section{BROWN. UNIV́ERSITY.}

Lulu B. Joslin.

AMCEBA BLATTE AND AMCEBOID MOTION.

THE writer wishes to call the attention of teachers of biology to a form of $A$ moeba that hitherto has been somewhat neglected in this country, but which is of much theoretical interest. It is, moreover, sufficiently plentiful and easily obtained at all seasons of the year to be adapted to the uses of small laboratory classes. This is Amoeba blatto Bütschli, which inhabits the intestine of the croton bug, or common cockroach, Blatta (Phyllodromia) germanica, a well-known immigrant from Europe that has established itself in our larger towns and, at least in the eastern states, in many country villages. Throughout the year this cockroach is active in bake-shops, creameries, sugar refineries and in the kitchens and basements of hotels, restaurants and private houses, where it may be found under sinks, about water pipes and in similar warm, dark places.

Rhumbler,' in a recent paper in the 'Festschrift' commemorating Professor Ernst Ehler's seventieth anniversary, describes fully the movements of this Amoeba, as well as the methods that have been employed at Göttingen for obtaining it. The cockroaches are etherized, the heads and terminal segments of the abdomen clipped off, and the intestine care-

\footnotetext{
${ }^{1}$ Rhumbler, L., '05, 'Zur Theorie der Oberflächenkräfte der Amöben,' Z. f. wiss. Zool., 83 Bd., pp. 1-52.
} 
fully pulled out with a pair of fine forceps into a few drops of one-half-per-cent. solution of common salt upon a glass slide. The intestine is then opened, and the contents scraped out and examined.

A preliminary attempt by the writer and his assistants to use Amoba blattce as a laboratory subject with a class of a hundred and forty beginners did not meet with success, but little difficulty has been experienced in demonstrating it to small groups of students. Usually at least one in every four or five of the cockroaches which we have examined this winter has proved to be abundantly infested with the amœba.

Amoba blattce is a rapidly moving organism, without pseudopods, and is of especial interest from the fact, which Rhumbler has demonstrated and which I have been able repeatedly to confirm, that backward peripheral currents occur in the endoplasm in addition to the axial forward stream.

The currents resemble those of a drop of oil or of other non-living fluid, in which the surface tension is unequally distributed. Such a drop contains an axial current, which flows in the direction of a certain area upon its surface over which the surface tension is diminished, turns outward in front, and forms backward superficial currents like a fountain. Hence the term 'fountain currents' has been applied to this phenomenon. In A. blattoe the backward superficial currents usually do not come to rest, as in the simpler fountain currents, but unite at the rear and enter the central forward stream, establishing thus that which Rhumbler calls a 'fountain whirl' ('Fontänenwirbel'). It is on the existence of such currents as these in Amoba and Pelomyxa that Bütschli, Rhumbler and earlier writers have based the theory that amoboid movement is due to diminished tension over a certain area of the surface, since drops of non-living fluid, e. g., oil or chloroform, submerged in water and acted upon in such wise that the tension over a certain area of the surface is suddenly diminished, flow in precisely the manner above described.
The reader will recall that Jennings, ${ }^{2}$ in his recent paper on the 'Movements and Reactions of Amoba,' denies the existence of 'fountain currents.' His careful and longcontinued studies were based, however, principally on $A$. limax, $A$. proteus, $A$. angulata and $A$. verrucosa, and did not include $A$. blattoe or members of the genus Pelomyxa, in which such currents have been found by other observers.

Locomotion in Amoeba verrucosa, and in the other species which came under Jennings's observation, is a rolling forward, as of a bladder half filled with water, or of a bag half full of shot, that is pushed over a flat surface. In this case the entire endoplasm continually streams forward, and the ectoplasm rolls onward around the endoplasm. A particle of soot, caught upon the surface of the advancing $A m c b a$, is carried forward until it reaches the anterior edge of the animal, then downward beneath the body, where it remains quiet until the $A m o b a$ has passed completely over it, and it lies beneath the posterior edge. It is then carried upward and again forward to the anterior margin.

Rhumbler concedes that this form of locomotion can not be due to inequality in ordinary surface tension, and attempts to explain it as the result of unequal pressure at different parts of the surface, which is brought about by the unequal contraction of the ectoplasm as the result of gelatinization. The endoplasm at the anterior end of an $A$ moba in motion, according to this view, meeting there less resistance than at other points from the pressure of the gelatinizing and contracting ectoplasm, tends to burst forth from its confining envelope, and to become transformed into ectoplasm. Elsewhere, particularly at the posterior end of the organism, the reverse is supposed to take place, viz., ectoplasm is converted into endoplasm. An explanation of the lessened resistance of the ectoplasm at the anterior margin is sought in the stretching of the outer layer in that region, brought about

2 Jennings, H. S., '04, 'Contributions to the Study of the Behavior of lower Organisms,' Carnegie Institution, Washington. 
by the friction produced by adhesion to the underlying surface, which acts in opposition to the forward pressure of the endoplasm.

This theory is based mainly upon the analogous behavior of drops of chloroform which creep or roll spontaneously over a thin coating of shellac, with which the bottom of a glass dish is covered. After the shellac is dry, water is placed in the dish, and drops of chloroform are introduced with a pipette. A drop, under such conditions, rolls spontaneously in the opposite direction from the side which first shrinks back from the surrounding shellac. The adhesion between one side of the drop of chloroform and the shellac results in dissolving away the latter, in the diminution of surface tension over this side of the drop, and a rolling motion in this direction. Thus the drop plows a path through the shellac, propelled onward by the diminution of surface tension at its forward margin, which in the drop is produced by adhesion to the shellac. The drop of chloroform soon contains shellac in solution which, under the influence of the surrounding water, stiffens into a 'gel,' the contraction of which is supposed gradually to replace surface tension, as the active agency which propels the drop. If the gelatinous coating is of unequal thickness and strength at different points, motion occurs in the direction of a thin, weak point, where the contents burst through the superficial envelope.

By this analogy Rhumbler finds support for the theory, long since put forth by Berthold, that amœboid motion, at least in the amœbæ without backward currents, depends upon a one-sided adhesion to the substratum, and also for his own idea that the rolling motion of such amœbæ is due to the centripetal pressure of the gelatinizing ectoplasm and the bursting forth of the endoplasm at the anterior end, which continues the adhesion to the substratum and forms new ectoplasm. The analogy illustrates the possibility also that a gradual transition may occur ontogenetically and phylogenetically between the method of locomotion in amœer with fountain currents and active interchange between endoplasm and ectoplasm, and that in others, like $A$. verru- cosa, with 'gelatinous' ectoplasm, which move without backward currents. Such a transition would depend upon the degree of gelatinization that had been reached. This process might be supposed to take place in A. blattce, for example, until the backward peripheral flow should cease entirely, and the gelatinizing ectoplasm should reverse the direction of its movement over its free surface, and turn forward.

It is evident that Rhumbler's theory rests largely on an analogy with the behavior of drops of non-living fluid under special conditions. Facts which indicate that a constant gelatinization and the opposite are actually taking place in amœbæ with rolling motions are extremely meager. It seems highly probable that $A$. blattoe moves in response to unequal surface tension; but Rhumbler's explanation of the more complicated (rolling) motions of other amœbæ, though a useful hypothesis, needs the support of more facts drawn from direct observation of the organism itself, before it can be proved.

Jennings describes the rolling movement as consisting of the outflow of a wave of protoplasm at the anterior end of the Amoba, which exerts a pull upon the upper surface, drawing it forward, while the hinder portion of the Amoba becomes released from the substratum and contracts. Thus the 'strong pull from in front and the slight contraction from behind' cause the posterior end, and the inner contents, to flow forward. Jennings, accordingly, places emphasis upon the pull from in front, and looks upon it as a more important factor in locomotion than the contraction of the ectoplasm at the rear.

Rhumbler's theory at this point departs from Jennings's interpretation of the facts. The supposed gelatinizing, or semigelatinous, covering of Amœba, according to Rhumbler, is constantly bursting at its anterior edge, which is attached to the substratum. Out of the breach the endoplasm flows forward, as the result of the contraction of the gelatinous covering of the upper and posterior surfaces. If these are the facts, there can be no strong superficial pull exerted from in front back- 
ward such as Jennings describes, for, according to Rhumbler, the ectoplasm in front is stretched between the vis a tergo and the friction against the substratum, is weakened and broken. The upper ectoplasm with a broken front edge can hardly be imagined to pull strongly upon the body behind it.

It is not expedient in this article to go more fully into the facts connected with this familiar phenomenon, which appears to be by no means as simple an action as Rhumbler supposes, when he compares it to the rolling of a rubber tire by hand or to the creeping of a drop of chloroform over a shellac-covered surface. Both of the papers cited, however, deserve the careful attention of every teacher of biology who touches upon the subject of $A m o b a$ and amoboid motion, although the conclusions of neither writer can be accepted without some modification.

\section{John H. Gerould.}

\section{A CULTURE MEDIUM FOR THE ZYGOSPORES OF MUCOR STOLONIFER.}

IN the first edition of his 'Methods in Plant Histology' Professor Chamberlain speaks of the zygosporic phase of Mucor as being ' rarely seen' and requests information of anyone obtaining it. In the recent edition of the same work he refers to the researches of Dr. Blakeslee and then gives directions for making cultures for the zygosporic stage. The method described is rather haphazard and the tone in which it is stated indicates that the results would be doubtful.

During the past three months the present writer has obtained the zygospores so frequently that he now feels confident of being able to secure them at any time within a week. With proper conditions of moisture and temperature, success is apparently dependent only on the nature of the substratum. The substratum used is corn muffin bread, made, according to the baker, after the following formula :

Corn meal .......... 16 pounds.

Flour ............. 3 pounds.

Lard .............. 3 pounds.

Salt $\ldots \ldots \ldots \ldots \ldots \ldots \ldots$. $1 / 2$ pound.
Eggs $\ldots \ldots \ldots \ldots \ldots \ldots \ldots \ldots 48$

Sweet milk .......... 3 gallons.

Baking powder ......... 18 ounces.

Half a dozen crumbs of this bread of the size of a thimble in as many tumblers, will yield on the average four or five cultures producing zygospores in large numbers in from five to seven days. The atmosphere should be kept saturated, the temperature about $70^{\circ}$ F. and darkness is favorable though not necessary.

A series of experiments have been made and others are now under way to determine more exactly the conditions of zygospore formation.

\section{College Park, Va.}

\section{J. I. HaMaker.}

\section{THE EFFECT OF FERTILIZERS ON THE REACTION} of solLs. ${ }^{1}$

THE effect of fertilizers on the reaction of the soil has interested both the farmer and the scientist for many years, but little experimental work appears to have been done on the problem, however. It is frequently held by farmers that the continued use of fertilizers, particularly of acid phosphates, and also potash salts and ammonium sulphate results in the failure of the red clover crop, a result which is attributed to the acid residues left in the soil by the selective action of plants in removing the essential elements from the salts in which they are applied. While there can be no doubt that certain fertilizing materials, notably ammonium sulphate, will produce an injurious degree of acidity, even changing the reaction of an alkaline soil, the evidence with regard to other fertilizers is not so positive.

Only recently have methods giving definite results been devised by which the total acidity of a soil may be determined. It is possible to determine the acidity of soil within practical limits by the lime-water method, ${ }^{2}$ and I have determined the present acidity of a known naturally acid soil which has received different fertilizing treatment, by this method.

Dr. Thorne, of the Ohio Experiment Sta-

\footnotetext{
${ }^{1}$ Published by permission of the Secretary of Agriculture.

${ }^{2}$ Jour. Amer. Chem. Soc., 26 (1904), 637.
} 